\title{
Effect of glycaemic index on body weight
}

\author{
Mette Axelsen
}

Department of Clinical Nutrition, Sahlgrenska Academy, Göteborg, Sweden

Abstract

Dietary carbohydrates result in different postprandial blood glucose and insulin responses, depending on the rate of digestion. The glycaemic index (GI) classification allows for ranking foods according to their effect on the glycaemic response: a high glucose response (high GI) or low glucose response (low GI). There has been great interest in recent years in whether low-GI foods are more satiating and lead to lower body weight than high-GI foods when incorporated into whole diets. Only a few studies with similar macronutrient and fibre intake have assessed the effect of GI on body weight. These studies, ranging from 5 to 16 weeks, have not been able to show improved satiation or lower energy intake or body weight. However, in one study the interesting observation was made that total fat mass was decreased. Further long-term studies using adequate differences in GI are needed to determine whether low-GI diets can beneficially affect long-term body weight homoeostasis in humans.

Keywords: energy intake; fat mass; glucose; glycaemic index; insulin; obesity

\section{Introduction}

Carbohydrates (starches and sugars) play an important role in human nutrition by providing energy to cells in the body, particularly the brain. The average minimum amount of glucose needed for normal brain function is $130 \mathrm{~g}$ per day. This level of intake, however, is typically exceeded to meet the energy and nutrient needs while consuming acceptable levels of protein and fat. The median intake of carbohydrates is approximately $200-300$ g per day for men and 180-230 g per day for women. At these levels of intake, carbohydrates may contribute to significantly elevated glycaemic and insulinaemic responses over the day. It has been postulated that the habit of consuming carbohydrates from refined bread and cereal products thus promotes the cluster of diseases associated with the metabolic syndrome, such as obesity and dyslipidaemia $(1,2)$. This has attracted vast academic and clinical interest and also led to numerous popular diet books.

\section{The glycaemic index}

The glycaemic index (GI) is the indexing of the blood glucose response of $50 \mathrm{~g}$ of available carbohydrate from a test food, compared with $50 \mathrm{~g}$ of carbohydrate from a standard food (white bread or glucose) (3). The index is based on measurement during a period of $2 \mathrm{~h}$. Determining the GI of carbohydrate-rich foods allows for ranking the glycaemic responses of various foods and, thus, separating those producing high glycaemic responses (high GI) from those producing a more modest blood glucose rise (low GI). In general, the insulin responses, when measured, relate well to glycaemic responses (4). It also appears that the rate of digestion of the food in in vitro analyses can be used as a proxy for measuring GI in vivo $(5,6)$. More slowly absorbed (low-GI) starchy foods are often less processed, or processed in traditional ways, such as wholegrain pumpernickel breads, cracked wheat or barley, brown rice, beans and lentils. The glycaemic response may also be reduced by adding certain acids or soluble fibre, by replacing high amylopectin starch with high amylase starch or by the addition of fat. Further, minimizing the degree of cooking or mincing often leads to a lower glycaemic response to starchy foods.

\section{The hypotheses}

Two main hypotheses for a potential detrimental effect of high-GI starchy foods on body weight and body composition have been commonly cited in the literature.

First, high-GI starchy foods could lead to an expansion of body fat and reduction in muscle tissue (7). This would result from both early and late events in the postprandial phase. In the early phase (0.5-1.5 $\mathrm{h}$ after ingestion), the prompt peak in insulin will promote carbohydrate oxidation and suppress fat oxidation. It has also been postulated 
to promote conversion of carbohydrates to fat. In the late phase ( $2-4 \mathrm{~h}$ after ingestion), the insulin level becomes low owing to a shortage of glucose from the intestine. Further, the preceding hyperinsulinaemia may result in undershoot in the blood glucose concentration to below its baseline. These changes, if present, may trigger increased cortisol and noradrenaline release, and gluconeogenesis from certain amino acids, and thus promote proteolysis (muscle breakdown).

Secondly, high-GI starchy foods could increase appetite and thus lead to passive overconsumption and obesity $(1,8)$. This has been proposed to result from elevated noradrenaline concentrations between meals (a potent appetite stimulus), decreased fullness due to faster passage of food from stomach to intestine and shortened time for satiety peptides in the gut to be released. Possibly, in addition, the utilization of carbohydrates and protein may be a signal to increase the stores of these fuels (i.e. stimulate overall food intake), since the body has a limited storage capacity for these macronutrients compared with fat.

\section{The evidence}

A closer look at the evidence shows that these hypotheses, at present, have several deficits and need to be revisited. In rats, consumption of low-GI food increases fatty-acid synthase activity in fat and promotes glucose incorporation into lipids (9). In one study, body fat was $71 \%$ lower and muscle mass $8 \%$ higher in rats given low-GI food (9). However, the lipogenic capacity of adipose tissue is substantially lower in humans than in rats, in part as a result of the lower abundance of sterol regulatory element binding protein 1c (SREBP-1c) (10).

Several single-meal studies of appetite and GI have been performed in humans, but with inconsistent results $(1,11,12)$. One study using preloads with varying GI found that carbohydrates with a high GI suppress subjective appetite and food intake in the short term, but that those with a low GI do not (13).

A study of overweight children found significantly greater weight loss after approximately 4 months of intervention in a paediatric obesity programme. However, in that study many other factors besides the GI varied between diet groups (14). A 2 week cross-over study with 18 normalweight women showed a lower ad libitum energy intake and weight loss with a high-GI diet than with a low-GI diet (15). However, in that study the highGI diet had a higher fibre content than did the lowGI diet. Further, a 4 month study of 24 subjects with impaired glucose tolerance (16) found a significantly smaller weight loss after a low-GI $(0.2 \mathrm{~kg})$ diet than after a high-GI diet $(0.5 \mathrm{~kg})$. That study substantially increased fibre intake in the low-GI diet, but the difference in GI during the study was extremely modest (high-GI: 59; lowGI: 54).

A 5 week cross-over study examined low versus high GI in 11 healthy overweight men (17). The lowGI diet had a $40 \%$ lower GI, but also a 50\% higher fibre content. In the period between 8.00 am and $4.00 \mathrm{pm}$ the insulin level was significantly lower at noon only on the low-GI diet. Moreover, on the low-GI diet, the glucose levels were higher, but the triglyceride levels lower, after lunch. Further, no significant effect on ad libitum energy intake or total body weight was detected at the end of the trial. However, that study also determined body composition by use of dual-energy X-ray absorptiometry (DEXA) and found a significantly reduced fat mass, by $0.7 \mathrm{~kg}$. The decrease in fat mass was accompanied by a decrease in leptin, lipoprotein lipase and hormone-sensitive lipase messenger RNA.

The most well-controlled intervention study exploring the effect of high versus low GI on ad libitum energy intake and body weight was published in 2004 (18). In that study, Sloth et al. investigated 45 healthy overweight women in a 10 week cross-over study. The difference in GI between supplied test foods (approximately $75 \%$ of total carbohydrate intake) was $24 \%$. The high- and lowGI diets had identical composition, apart from the GI. At 10 weeks, the low-GI diet had resulted in higher fasting glucose but lower low-density lipoprotein-cholesterol concentrations compared with the high-GI diet. In contrast, the low-GI diet did not result in any significant difference in energy intake, body weight or fat mass assessed by DEXA. Even though subjects in the low-GI group lost a total of $1.9 \mathrm{~kg}$, compared with $1.3 \mathrm{~kg}$ lost by the high-GI group, this difference was not significant. With such a modest difference between diets, more study power in the form of an increased number of subjects or greater difference in GI than used in this study would probably be needed to detect significant differences between groups. One study in humans found a negative nitrogen balance with consumption of a high-GI diet compared with a 
low-GI diet (7), suggesting that high-GI diets may lead to catabolism of lean body mass. In contrast, the study by Sloth et al. (18) found a more negative nitrogen balance with consumption of a low-GI diet. No study has found an effect on direct measures of lean body mass.

\section{Conclusions}

In summary, a low GI is beneficial in terms of blood lipid lowering and may have several other beneficial metabolic effects in subjects at risk of the metabolic syndrome (19). However, at present the compiled data are not in favour of the contention that low-GI diets are more satiating, result in lower ad libitum energy intake or reduce body weight compared with high-GI diets with similar fibre and macronutrient contents. In addition, inconsistent results have been obtained in terms of body composition. Further long-term studies are needed to delineate whether adequately lowered GI can beneficially affect lipid homoeostasis in humans, such as transcription of genes involved in metabolic conversion of glucose to fats, postprandial concentrations of lipoproteins, long-term body weight homoeostasis and central obesity.

\section{References}

1. Roberts SB. High-glycemic index foods, hunger, and obesity: is there a connection? Nutr Rev 2000; 58: $163-$ 9.

2. Pawlak DB, Ebbeling CB, Ludwig DS. Should obese patients be counselled to follow a low-glycaemic index diet? Yes. Obes Rev 2002; 3: 235-43.

3. Jenkins DJ, Wolever TM, Taylor RH, Barker H, Fielden $\mathrm{H}$, Baldwin JM, et al. Glycemic index of foods: a physiological basis for carbohydrate exchange. Am J Clin Nutr 1981; 34: 362-6.

4. Wolever TM, Bolognesi C. Source and amount of carbohydrate affect postprandial glucose and insulin in normal subjects. J Nutr 1996; 126: 2798-806.

5. Granfeldt Y, Bjorck I, Drews A, Tovar J. An in vitro procedure based on chewing to predict metabolic response to starch in cereal and legume products. Eur J Clin Nutr 1992; 46: 649-60.

6. Englyst KN, Vinoy S, Englyst HN, Lang V. Glycaemic index of cereal products explained by their content of rapidly and slowly available glucose. Br J Nutr 2003; 89: 329-40.

7. Agus MS, Swain JF, Larson CL, Eckert EA, Ludwig DS. Dietary composition and physiologic adaptations to energy restriction. Am J Clin Nutr 2000; 71: 901-7.
8. Ludwig DS, Majzoub JA, Al-Zahrani A, Dallal GE, Blanco I, Roberts SB. High glycemic index foods, overeating, and obesity. Pediatrics 1999; 103: E26.

9. Pawlak DB, Kushner JA, Ludwig DS. Effects of dietary glycaemic index on adiposity, glucose homoeostasis, and plasma lipids in animals. Lancet 2004; 364: 778-85.

10. Letexier D, Pinteur C, Large V, Frering V, Beylot M. Comparison of the expression and activity of the lipogenic pathway in human and rat adipose tissue. $\mathbf{J}$ Lipid Res 2003; 44: 2127-34.

11. Ludwig DS. Dietary glycemic index and obesity. J Nutr 2000; 130: 280.

12. Raben A. Should obese patients be counselled to follow a low-glycaemic index diet? No. Obes Rev 2002; 3: $245-$ 56.

13. Anderson GH, Catherine NLA, Woodend DM, Wolever TMS. Inverse association between the effect of carbohydrates on blood glucose and subsequent short-term food intake in young men. Am J Clin Nutr 2002; 76: 1023-30.

14. Spieth LE, Harnish JD, Lenders CM, Raezer LB, Pereira MA, Hangen SJ, et al. A low-glycemic index diet in the treatment of pediatric obesity. Arch Pediatr Adolesc Med 2000; 154: 947-51.

15. Raben A, Holst JJ, Madsen J, Astrup A. Diurnal metabolic profiles after $14 \mathrm{~d}$ of an ad libitum highstarch, high-sucrose, or high-fat diet in normal-weight never-obese and postobese women. Am J Clin Nutr 2001; 73: 177-89.

16. Wolever TM, Mehling C. High-carbohydrate-low-glycaemic index dietary advice improves glucose disposition index in subjects with impaired glucose tolerance. Br J Nutr 2002; 87: 477-87.

17. Bouche C, Rizkalla SW, Luo J, Vidal H, Veronese A, Pacher N, et al. Five-week, low-glycemic index diet decreases total fat mass and improves plasma lipid profile in moderately overweight nondiabetic men. Diabetes Care 2002; 25: 822-8.

18. Sloth B, Krog-Mikkelsen I, Flint A, Tetens I, Bjorck I, Vinoy $\mathrm{S}$, et al. No difference in body weight decrease between a low-glycemic-index and a high-glycemic-index diet but reduced LDL cholesterol after 10-wk ad libitum intake of the low-glycemic-index diet. Am J Clin Nutr 2004; 80: 337-47.

19. Jenkins DJ, Kendall CW, Augustin LS, Franceschi S, Hamidi M, Marchie A, et al. Glycemic index: overview of implications in health and disease. Am J Clin Nutr 2002; 76: 266-73S.

\section{Mette Axelsen}

Department of Clinical Nutrition

Sahlgrenska Academy

PO Box 459, SE-405 30, Göteborg

Sweden

E-mail: mette.axelsen@nutrition.gu.se 